\title{
Transforming the Workplace: Unified Communications \& Collaboration Usage Patterns in a Large Automotive Manufacturer
}

\author{
Anthony Bolton \\ Global Information Technology \\ General Motors \\ Anthony.Bolton@gm.com
}

\author{
Meg Murray \\ Coles College of Business \\ Kennesaw State University \\ mcmurray@kennesaw.edu
}

\author{
Joy Fluker \\ Global Information Technology \\ General Motors \\ Joy.Fluker@gm.com
}

\begin{abstract}
The business communications landscape continues to change dramatically as a plethora of communications channels and devices become available and mature. Unified Communications and Collaboration (UC\&C), the integration of multiple communication channels and collaboration technologies, represents innovation in the communications technology space. The promise of $U C \& C$ is that it will increase productivity, spur innovation, and improve collaboration by providing an infrastructure that supports the rising knowledge economy, the digitally connected enterprise, and the virtual work environment. This study examines the usage patterns of employees following the implementation of a UC\&C platform within a large automotive manufacturer, namely General Motors. The results indicate that UC\&C has had a positive impact on both business and employees and offers the potential to leverage the benefits of rich collaboration, interactive experience, and human intelligence to transform the workplace.
\end{abstract}

\section{Introduction}

Communication and collaboration have always been hallmarks of a successful business. Fueled by advances in communications and information technology, the landscape of business communication has changed dramatically over the years. As noted by Burg [1], most business no longer occurs face-to-face but instead occurs over communications mediums such as conference calls and email chains. The problem with technology-mediated communications, however, has been that it introduces an artificial intermediary into the communication process. Consequently, while business is dependent on communications technology, user dissatisfaction is commonplace. According to Burg [1], the answer to this dilemma is not abandoning technology "for a more human way of working, but to evolve technology to make the new way of working more human" (p. 1).

Unified Communications and Collaboration (UC\&C) represents innovation in the communications technology space. UC\&C is positioned as an enterprise level solution that integrates heterogeneous communications channels and collaboration technologies to facilitate the interactive use of multiple communication methods and information sharing activities [2]. Most UC\&C solutions provide singleplatform support for telephony, audio, chat (text messaging), video, file and application sharing, presence management and mobile interaction. Often cited as a major strength of these technologies is the incorporation of features that include contextual presence tracking providing information about individual availability and communication method preference, on-demand switching between communications channels, and intelligent notification services [3]. But UC\&C is not about a checklist of features; it is about creating a holistic and dynamic approach to an organizational communication strategy that spans people, processes, and technology. The primary goal of unified communications is to improve user productivity and enhance business processes [2]. The organizational need for a unified communications strategy is more pronounced as the constraints of time and place become irrelevant within a more global and virtual business environment operating in an economy increasingly dependent on real-time information flow and knowledge exchange.

To remain viable in today's fast-paced business world, companies are driven to innovate even if that means embracing technologies and trends that promise to be disruptive and transformative. The expectation is that UC\&C will transform the workplace by providing a more synchronized fit between the way people communicate in the workplace and the technology they use. The promise of $\mathrm{UC} \& \mathrm{C}$ is that it will increase employee productivity via streamlined business 
workflows, improve collaboration between employees, business partners and customers, and spur innovation by fostering knowledge sharing and knowledge transfer [4]. The adoption of UC\&C is growing rapidly. According to market research, the UC market will reach $\$ 75.81$ billion [increase of $16.3 \%$ ] by 2020 [5]. However, empirical research related to $\mathrm{UC} \& \mathrm{C}$ is scarce. The objective of this study is to analyze the adoption and usage patterns of UC\&C implementation in a major automotive manufacturer, namely General Motors, and examine its potential to transform the workplace.

\section{Drivers for Unified Communications and Technology}

Several factors are influencing the demand for enterprise-wide communications strategies that facilitate collaboration and connect people in more efficient and intuitive ways. In the age of the knowledge economy, the forces of globalization and digitization are leading to transformation of the business environment. According to the McKinsey Global Institute, the past decade has witnessed a shift to a digital knowledge economy where knowledge has taken precedence over physical labor within the enterprise [6]. Knowledge has become a core business asset and knowledge exchange a contributing factor to business success [7]. Consequently, the development of communication systems to aid in the effective sharing and leveraging of that knowledge has become a vital component of enterprise strategy [8] and studies have confirmed that the ability to share knowledge through information technology tools improves business performance [9]. Further, the knowledge economy has given rise to decentralized organizational structures leading to increasingly virtualized work environments that rely heavily on virtual teams to accomplish organizational objectives [10]. Collaborative technologies provide powerful support for making effective global virtual teams a reality [11].

At the same time, the 'digitization of everything' craze is sweeping across all industries and is poised to reshape modern business [12]. Companies are compelled to embrace digitization as a way to transform a business through differentiation, increased productivity, improved internal and external relationship-building, support for real-time business decision-making, and a heightened focus on innovation. The process of transformation and digitization within an enterprise, however, results in a reciprocal increase in requirements for connectivity and communication between people, digital devices, and information and communication technology systems. Unified communication and collaboration systems provide the pathways for the dissemination of knowledge, facilitation of decision making, and seamless connection of people within the broader digitally connected world [13].

Juxtaposed to the demands of the new work environment, the new communication mediums are actually making the communication process more cumbersome and complex [14, 15]. Communicators have the added work of managing a myriad of devices as well as determining the optimal medium for a given situation [14]. No longer are the choices simply to pick up the telephone or schedule a face-to-face meeting. Additional options are now available such as video calling and chat sessions. Many of these options are provided using different and siloed solutions. UC\&C offers an integrated and uniform communications infrastructure, but the key to successful adoption will be predicated on its acceptance and proficiency of use. Research has shown that factors such as organizational culture can impact the effective adoption of communications and collaboration technologies [16] as can challenges in the areas of technology [17] and process. Research carried out by Pickering and Wynn [18] across Intel's virtual workplace suggested that customization of the collaboration platform and architecture aligned with particular business processes versus strict delivery of feature capability (one size fits all) is required to optimize results within enterprise-scale environments. In short, a common -- but simplified-- user experience supported by an organizational culture that is flexible and adaptable purports the most promise for a successful implementation of UC\&C [16].

Communication technology and services, such as UC\&C, will play an increasingly important role as people adapt to the evolving digital world through which they communicate and collaborate. The necessity of UC\&C in the workplace has been widely acclaimed by industry research groups such as Gartner and by anecdotal case studies by vendors. According to Greenard of CIO Insight [19], UC\&C has moved from a cost-savings venture into a mission critical strategy. UC\&C promises to increase productivity, heighten collaboration, and improve business processes by reducing overlapping work, spurring innovation, speeding up development cycles, and improving service levels [19]. UC\&C provides an infrastructure poised to address the needs of the new knowledge economy, the digitally connected enterprise, and the virtual work environment. So far, however, clear expectations of exactly what UC\&C delivers beyond an enhanced communications system have yet to be clarified [20]. UC\&C is not about a set of tools; it is 
about a new way of working and UC\&C is evolving in ways no one could have predicted [20].

\section{The Study}

This study examined the adoption and usage patterns of employees following the implementation of a Unified Communications and Collaboration platform (Microsoft's Skype for Business) within a large automotive manufacturer (General Motors). Skype for Business, formerly known as Microsoft Lync, is an enterprise-based communications system solution that integrates audio and video conferencing, click-to-talk, pc phone, and chat [instant messaging], with services such as presence management, file sharing, and application (app) sharing. Previous communications support at General Motors included disparate telephone, messaging, and conferencing systems.

General Motors was founded in 1908 and today is one the world's largest multinational enterprises, ranking five in the Fortune 500 and 21 in the Global 500. GM employs over 206,000 people, spanning six continents with more than 390 corporate facilities. GM is the third largest automotive manufacturer as measured by revenue. GM manufactures vehicles in over 70 manufacturing plants in 37 countries. General motors core brands include Chevrolet, Buick, Cadillac, Holden, HSV, Wuling, Baojun, Jie Fang, UzDaewoo, Alpheon, Opel and Vauxhaul. GM supports a retail network of over 21,000 global dealerships, selling 9.9 million vehicles worldwide in 2015 earning revenue of $\$ 152$ billion.

UC\&C was rolled out to $100 \%$ of GM's full time salaried employees housed within GM's four primary business function units including global corporate, sales, marketing, product design, product development and connected customer experience. This represents approximately 120,000 individuals. The rollout occurred in phases. From January to June 2015, UC\&C was only enabled in those units that provided IT and telecommunications support. Beginning in July 2015, UC\&C was made available to all employees; however this followed a four-month long targeted deployment strategy based on user location and readiness. GM enacted several initiatives to increase employee awareness of the UC\&C program and to encourage adoption. Regional advocates were solicited, trained, and assigned to meet with business groups to provide demonstrations on the ease-of-use of the new system. Booths were setup at main corporate offices and manned with greeters adorned in 'Skype for Business' T-shirts. A web portal that included helpful information and provided online training was made available. Lunch and Learns were provided at various global locations to introduce the technology and highlight its benefits. In addition, employees had the option of requesting in-person training sessions. It should be noted that formal training was not required. The expectation was that employees already had familiarity with the various communications tools incorporated into the UC\&C platform so the cost of implementing a formal training program would not be necessary.

During the transition period, UC\&C ran in parallel with the existing system. In October 2015, the legacy system was disabled thereby eliminating the previous softphone capabilities and messaging support. A streamlined version of the legacy audio/video conferencing system was retained. This study reports monthly UC\&C usage data from October 2015 through April 2016. The data was collected real-time by the UC\&C reporting capability.

\section{Findings}

Adoption of the UC\&C system was voluntary. Adoption patterns were tracked by user logons to the system. The number of users was measured by unique usernames while system interaction was measured by individual user logon sessions. As seen in Figure 1, the number of users and logon sessions increased over the seven-month period. There was a slight dip in December but this can be attributed to the holiday season that occurs during this month. (In fact, the use of all communications tools decreased during the month of December.) In October, there were 110,942 unique users accounting for approximately 488,000 sessions. By the end of April there were 122,333 users accounting for more than 603,000 sessions. The increase in system use far exceeded expectation. Prior to the rollout, Key Performance Indicators (KPIs) or metrics were determined to ascertain adoption goal achievement. A measure of $100 \%$ indicates attainment of the business objective. By mid-October the KPI metric exceeded $100 \%$ reaching $124 \%$ and by November it was $138 \%$. In fact, the UC\&C system was being more heavily used than the previously available communications tools. What this indicates is that the users saw the value of the UC\&C technology and quickly began using its functionality. This occurred without instituting a formal training program or mandatory use policy and while GM employees still had other communication channel options available. The key to successful adoption of UC\&C is predicated on its acceptance and proficiency of use. 


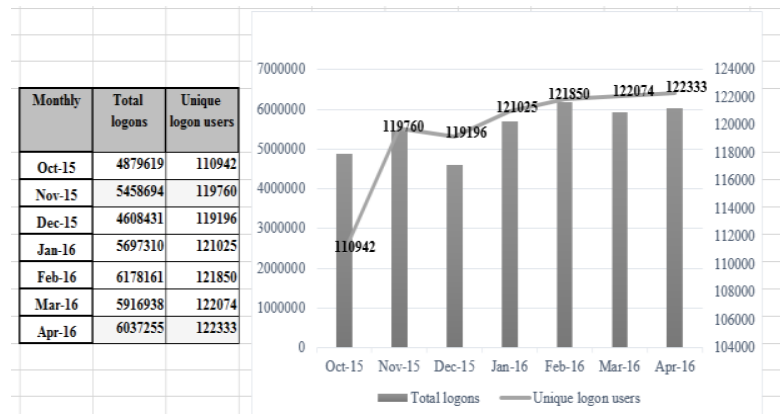

Figure 1. Number of users

UC\&C functionality included audio calls, video calls, instant messaging, application sharing and file transfer. Instant messaging was the most widely used tool. Large increases in the number of instant message sessions occurred as users transitioned to the UC\&C platform from the previously provided instant messaging system. Figure 2 shows data for total sessions, total number of messages and average number of messages per session. In general, each IM session averaged between 6 and 8 messages.

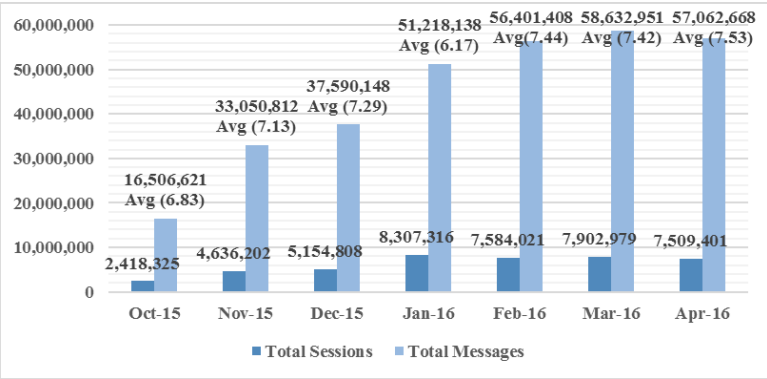

Figure 2. UC\&C instant messaging sessions

As shown in Figure 3, audio calls were the next most heavily used communications medium followed by the use of the file transfer feature. The benefit of the file transfer tool is that it allows files to be exchanged real-time even while engaged in an audio, video or instant messaging session. Video sessions were least frequently utilized.

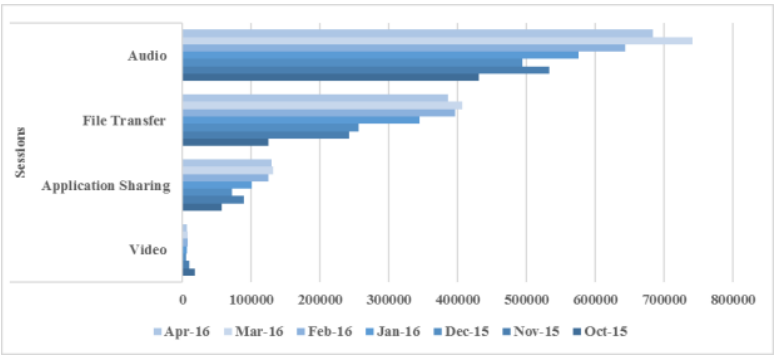

\section{Figure 3. UC\&C usage by feature}

Interestingly, usage metrics post deployment when compared to legacy system use in the prior year (from January to July) show a shift in overall volume and also a shift in service usage mix. As shown in Figure 4 , one of the major findings was that overall instant messaging volumes decreased. Volume usage in audio calls, video calls, and application sharing increased dramatically. The drop in instant messaging volumes can be explained by the increase in the use of other communication modalities. For example, fewer messages are being sent as users switch from chat to other modes of communication such as voice or video. Users are switching because the UC\&C system makes it trivial to move between the various forms of communications. Further, the decrease in use of chat can be also be explained by the increase in the use of application sharing. As compared to other mediums such as voice or video, communicating via chat while engaging in the review of shared material is very cumbersome.
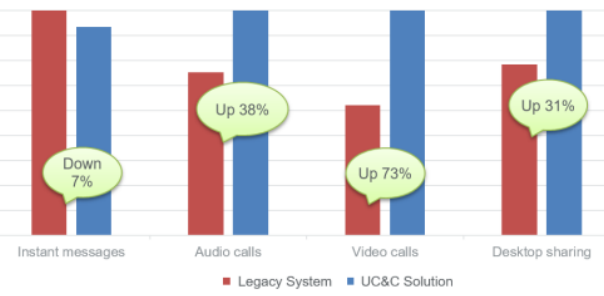

\section{Figure 4. Usage metrics UC\&C versus legacy system}

Audio and video calls can be distinguished by two categories, peer-to-peer conversations that consist of two participants and conferences that consist of three or more participants. As shown in Figure 5, peer-topeer audio conversations occurred quite frequently and increased over time. Even so, the average length of a call stayed consistent around 6 to 7 minutes. It should be noted that the integrated voice capability within the UC\&C system has proven effective in driving a shift to IP based softphone feature adoption. The legacy softphone client was limited in capability; for example, it was not possible to create or manage an ad hoc multi-point call. Phone functionality was limited to point-to-point single party calls. The phone client was also cumbersome to use and required specific attention to be paid to the configuration of headset and other adjunct input devices. Click-to-call was not widely used and the softphone functionally was mainly leveraged when individuals were travelling or working remotely. Finally, the softphone, while being used, had to remain connected to the GM network. The UC\&C client does not have these restrictions and is 
able to automatically authenticate to an externally exposed access server, thereby increasing ease of access. This also optimizes call quality for both voice and video. A significant increase in the voice use and adoption was experienced with the UC\&C system; in a yearly comparison, a $153 \%$ growth rate in active individual use of the voice feature over the legacy system was evidenced.

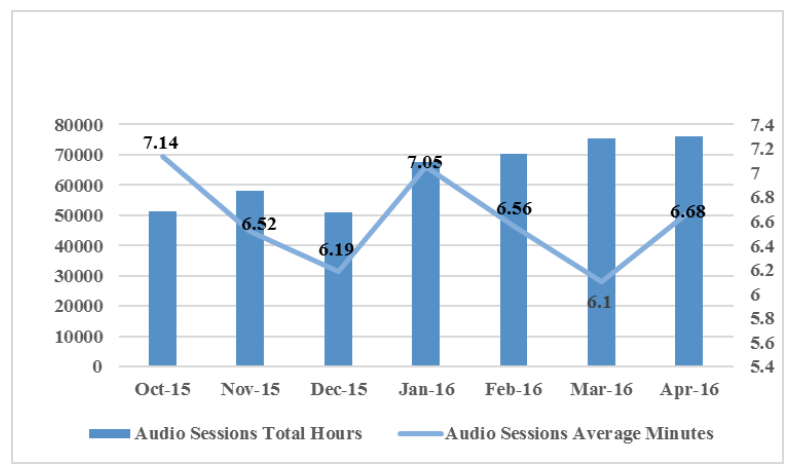

Figure 5. UC\&C peer-to-peer audio sessions

Video conversations represented the least frequently used communications medium within the UC\&C system. (Video conferencing - video call with more than two participants - is more prevalent.) The difference between peer-to-peer audio and video conversations is dramatic. For the period under review, the ratio of audio conversations to video conversations was 64:1. However, as shown in Figure 6 , the use of peer-to-peer video conversations increased over time as did their duration. Interestingly, the average duration of a video call was more than 2 minutes longer than an audio call. As noted before and indicated in preliminary post deployment end user surveys, the integrated functionality and intuitive operation of the UC\&C system make it easier for the user to leverage voice and video features. It also reduces the time it takes to access colleague contact data (via the context-based presence feature), initiate calls and also to escalate dynamically from one communication modality to another (i.e. from chat to voice or video). This has major implications for simplifying the communications landscape and for aligning the communications modality with the tasks at hand. The data suggests users are proactively choosing the communications medium they feel best meets the needs of the situation.

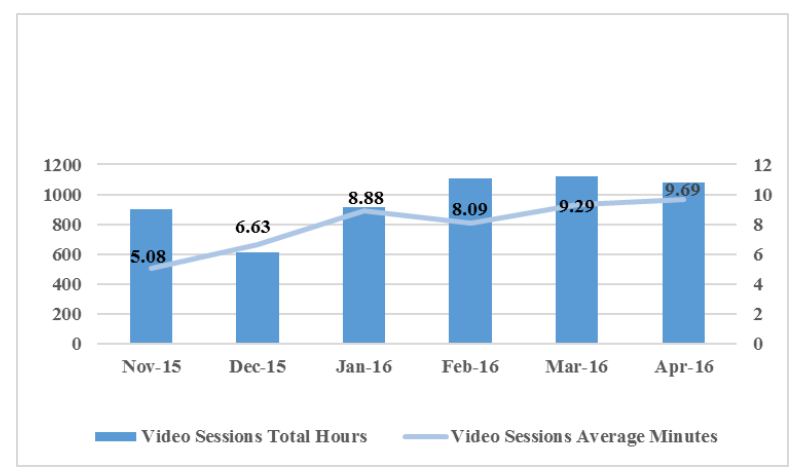

Figure 6. UC\&C peer-to-peer video sessions

In addition to the UC\&C platform, GM has retained the use of a legacy audio/video conferencing system. There are similarities between the systems, but there are also substantial differences. At the current time, the legacy tool is predominantly used for scheduled meetings and the UC\&C tool for ad hoc meetings. This is because the legacy system is integrated with Microsoft Outlook, the company-wide calendar, but not yet implemented in the UC\&C System. This means scheduling a meeting within $\mathrm{UC} \& \mathrm{C}$ requires a special invite to participants. Another major difference between the two systems is the ability to add meeting participants while a meeting is ongoing. The legacy system does allow participants to be added on an ad hoc basis; it requires additional steps. A link to the meeting must be generated and sent to the participant who then has to enter their user information before being allowed to join the conference. The UC\&C tool has the ability to create and conduct an ad hoc conference much more efficiently. Users may be added to ongoing meetings by simply clicking their name as it appears within a contact list associated with the UC\&C system. As presented in Figure 7, a comparison between the video-enabled ad hoc and scheduled conferences shows that scheduled meetings occur more frequently and thus the legacy system is used more often for video-enabled meetings with three or more participants. On average, there were 6151 conferences per month using the UC\&C platform and 9144 conferences via the legacy system. While this seems to be a large difference, the number of video conferences within UC\&C far exceeded expectations given that it was initially enabled only for internal meetings. Participant size was also different. The UC\&C conferences averaged three participants, the legacy system averaged five. Again, this suggests that employees are finding value in the ability to initiate ad hoc conferences indicating increases in knowledge sharing activities. Other benefits are realized through the reduction in time that it takes to set up a UC\&C conference that can be channeled into other tasks and 
the reduction in travel costs that results as video conferences replace the need for many physical faceto-face meetings.

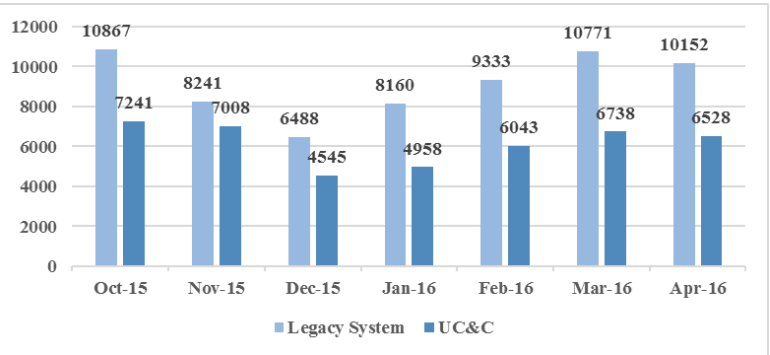

Figure 7. Video conference use: UC\&C versus legacy system

Meeting duration between the two systems was also compared. As shown in Figure 8, the number of minutes participants spent in a UC\&C conference was considerably less than the time spent in meetings conducted via the legacy system. More investigation is necessary to understand this trend. At the current time, the legacy system is preferred for scheduled meetings. However, the integrated features such as file transfer and the ease of use of application sharing within the UC\&C system help facilitate meeting agendas.

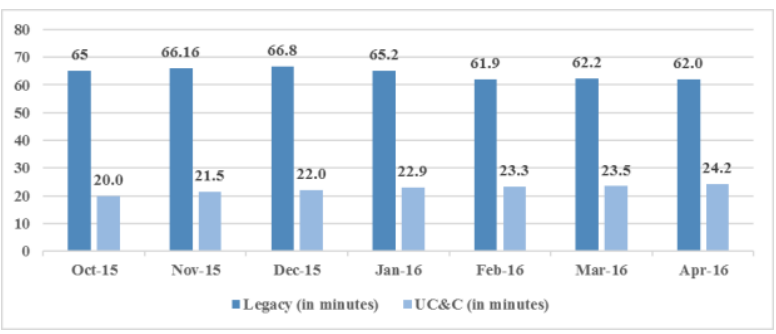

Figure 8. Average video conference duration: UC\&C versus the legacy system

App sharing, short for application sharing [also referred to as desktop sharing], allows meeting participants to share their desktop. Figure 9 shows data related to the use of app sharing between the two systems. App sharing occurs quite often during conference sessions. Notable are the trends lines for each platform. The use of app sharing in the legacy system is trending downwards while app sharing within the UC\&C platform is increasing.

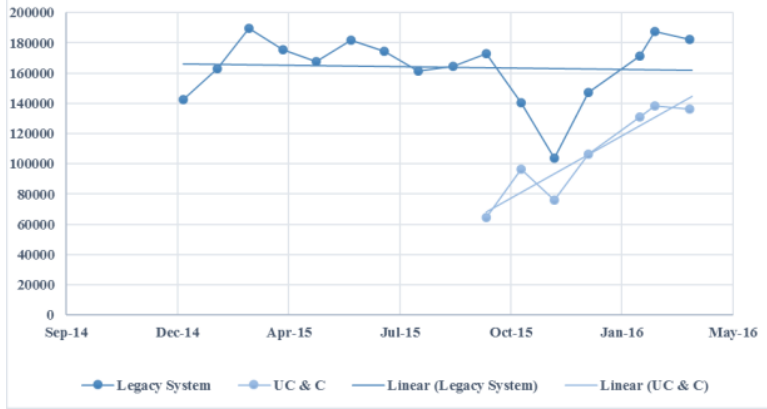

Figure 9. Trends in use of application sharing: UC\&C versus legacy system

The contrast between participant interchange within a meeting conducted via the legacy system and the $\mathrm{UC} \& \mathrm{C}$ system is stark. Prior to the implementation of UC\&C, users had to navigate a number of disparate systems in order to achieve their communication goals. An example of a simple workgroup meeting scenario best explains the differences. Within the legacy system, when three or more people wished to discuss and work on a presentation, they would have to (a) schedule a conference call to facilitate multi-party voice capability, (b) schedule a WebEx content sharing session integrating the voice conference bridge details, (c) dial the conference bridge and enter the bridge code, (d) manually connect to the conference application and enter their credentials, and (e) designate one individual to share a work product such as a PowerPoint presentation. In this scenario, three separate systems were required to facilitate the fundamental communication requirements, a legacy phone system or softphone, Intercall voice conference and Cisco's Webex application sharing solution. All three systems required pre-established user privileges, scheduling, and operation. If during review of a PowerPoint presentation, participants wanted to make a simple modification, for example adding a graphic developed by one of the team members, they would have to email the graphic file or store it offline where a designated editor would locate the file, open the graphic and incorporate it into the presentation; all done outside the purview of the conference participants.

The above scenario can be contrasted with a UC\&C experience where all functionality is integrated into a single system. The steps to achieve the same outcome as previously described would be (a) schedule a meeting or hold the meeting ad hoc, (b) have participants click on a meeting invite to join the collaboration session or in the case of an ad hoc meeting, have one individual simply drag an icon of 
each of the other participants into a live session window (c) assign a presenter to share the PowerPoint presentation (d) have the team member who developed the graphic simply click and share the image whereby the designated editor could then update the PowerPoint with the graphic in real time. All communication activity is achieved via one tool. No additional setup or credential verification is needed as participants are pre-verified before they logged into the system. Any modifications conference participants desired to make can be made 'on the fly' inclusive of file sharing. Further, with just a single click, an audio session can be escalated to full-featured video collaboration. The reduction in complexity reduces meeting time, increases meeting productivity, motivates employees to use the system, and creates a more natural way for technology-mediated collaboration to occur.

\section{Discussion of the Finding}

Prior to the deployment of GM's integrated UC\&C Client in October 2015, only 10,278 users within GM actively leveraged IP softphone [desktop] functionality. This number was a subset of 32,605 users configured for the legacy service. This indicates that user adoption and technical user acceptance was low with the legacy softphone technology. The legacy solution for softphone focused only on the delivery of voice and provided no additional functionality within the client such as instant messaging, video, file or content sharing. The application also provided limited quality for remote users as the laptop had to be connected via VPN to the corporate network. Finally the legacy softphone application was unavailable on all corporate mobile platforms (tablets \& phones).

Within the scope of the UC\&C deployment in GM, the 10,278 active users were converted to full softphone capability (inbound, outbound dialing with dedicated inbound number). The non-active legacy softphone users were required, along with other employees, to re-apply for softphone services. Within the first six months of deployment, an additional 10,082 requests were fulfilled and enabled with full UC\&C softphone functionality. Usage data shows that in May 2016, 21,496 users were actively using UC\&C softphone functionality, representing an over $100 \%$ increase in active use and adoption of the feature via UC\&C versus the legacy solution. The active voice users also exceeds the number of users configured for full voice feature services required for external calling. This shows that over 1,000 additional users, not configured for external dialing, are actively choosing to leverage the UC\&C voice services for internal collaboration. The UC\&C solution has resulted in a doubling of active use and over 1,000 unsolicited or trained (on UC voice functions) users choosing to voluntarily adopt.

Similar success has been seen in collaboration via desktop video. Year-on-year comparison of usage with the legacy hosted desktop video client vs. the UC\&C client shows a $75 \%$ increase in monthly use. Within 6 months of deployment, usage results show that over 2,700 users actively engage in video collaboration via the UC\&C client generating over 660,000 minutes of video collaboration per month. Video collaboration via the UC\&C client was enabled for all configured users, however, it was decided not to promote the feature or train users until late 2016. This is another example where users have chosen to proactively leverage UC\&C functionality, independent of specific training. It also validates the simple operation and effective interface provided by the UC\&C client, through facilitating user enabled adoption with minimum overhead in terms of training or end user complexity.

A high level of proactive user driven adoption has been observed with the UC\&C client deployment. Users have taken proactive steps to adopt and leverage advanced features ahead of scheduled feature deployment and training. An example of this is the leverage of Skype for ad hoc multi-party conferences. The Skype Unified Communications client provides a converged multi-mode and multi-media capability with features allowing for flexible escalation of sessions between different media modes (voice, video, chat). The Skype UC\&C client also allows ad hoc escalation from peer-to-peer sessions to multi-party, multi-media sessions. This was not possible with the legacy hosted client which was restricted to peer-to-peer sessions for voice and video only. Within the legacy system, establishment of a pre-scheduled meeting on the externally hosted collaboration system was required in order to facilitate a multi-party conference. While the official launch for UC\&C enabled multi-party conferencing is scheduled for later in 2016, the level of active ad hoc mixed voice/audio/messaging multiparty conferences already exceeds the legacy client video sessions by over $70 \%$.

Usage data for voice and video sessions on the unified client indicate a shift to ad hoc leverage of services. The legacy messaging solution required prescheduling of multi-party meetings. The UC\&C solution facilitates reservationless, ad hoc meeting establishment with the simultaneous leverage of voice, video, and messaging. In conjunction with a rise in ad hoc conferencing usage, data shows a significant reduction in average conference duration. The average duration of UC\&C multi-party conferences averaged 24 minutes versus the average traditional audio and 
web content sharing conference duration of 62 minutes. People are conferencing more frequently, leveraging multiple modes of media simultaneously, and using time more efficiently, reducing meeting times by on average $62 \%$ compared to scheduled meetings.

Post deployment impact of UC\&C within GM, leveraging system usage data, focused on the dimensions of adoption rate, proliferation of active use, and behavioral change. Adoption has been proactive characterized by a proliferation of active use. Overall, the utilization data supports a view of cultural and behavioral change in addition to technical and operational acceptance by the users. Prior to the implementation of $\mathrm{UC} \& \mathrm{C}$, the leverage of integrated multi-channel communication and computer mediated collaboration was relatively low when viewed in terms of usage as a percentage of the global worker population. Changes in modes of access are also occurring. Prior to the UC\&C deployment, corporate instant messaging, audio and video on mobile devices was practically zero. Since deployment, instant messaging via the UC\&C system (Skype Mobile) has grown by more than $16 \%$ and audio/video calls by more than $21 \%$. This aligns to increases in productivity as clearly these individuals are using their devices 'on the go', i.e., while traveling outside the office or even at home. The usage data reported in this study and the resulting analysis addresses the question of UC\&C adoption and acceptance suggesting it has had a positive impact on both business and employees.

\section{Conclusion}

The successful deployment of unified communications and collaboration within General Motors is a key technology enabler in the company's broader strategy for business digitization. Digitization of the business enterprise encompasses both transformation of legacy analogue processes and the expansion of business models to include integrated digitally supported products and services. UC\&C is one such service.

Within the context of this study, it is important to understand the associated GM business drivers aligned to digitization. Digitization is increasingly infiltrating the experience of GM's customers. GM is transforming into a technology rich company both from the perspectives of how it develops it products and in the manner that products themselves are increasingly being connected and enriched with digital technology. GM's digital transformation transcends all functions and lines of business and GM is quickly becoming a digital company with rich opportunity to expand its capability through digital strategies. For example, over the past three years, GM has strategically invested in the insourcing and convergence of its global Contact Centre infrastructure and applications. Through this program, GM has been able to enhance and develop its suite of applications and communication channels to its customers and dealers, introducing omni-channel experiences that enhance service delivery, performance, and ultimately customer experience. Further, GM has lead the automotive industry for over a decade in the field of connected vehicles through its OnStar business. GM is now expanding into other areas of digital service research, development, and service creation including autonomous and assisted driving, virtual reality design supporting marketing, ride sharing, and urban mobility applications. In the area of product development and manufacturing, GM is embracing the emerging Industry 4.0 and Industrial Internet revolution. Enabled via ubiquitous wired and wireless networking availability within its plants, GM has been an early adopter of the Internet of Things (IoT) models for proactive digital operations in manufacturing. GM has partnered with technology providers to implement an intelligent, cloud based IoT solution that facilitates proactive maintenance for critical plant machinery, optimizing production up-time.

GM views digital transformation as strategic to its continued growth and success, and digital technologies as the means to achieve strategic ends -- hallmarks of a digitally mature organization positioned to excel in the new economy [21]. UC\&C is central to GM's digital strategy. Process, technology, data and communication feature strongly within any digital strategy, however, people are essential within the process of transformation. People bring meaning to data and $\mathrm{UC} \& \mathrm{C}$ provides the mechanism to seamlessly integrate people with the digital landscape that includes systems, processes, and intelligence. Through a converged platform of heterogeneous networks, communication systems and feature applications, UC\&C technology serves to digitally integrate each and every individual within the enterprise, anytime, anywhere, any place, and on any device. UC\&C client and associated backend infrastructure provide a seamless panel through which key decision makers can connect to both human and digital information sources, collaborate, and effect critical change with velocity. Without human integration, any digital transformation is unlikely to succeed. By understanding this, GM, as an organization, is a visionary leader in the developing area of industrial and enterprise digital transformation.

The implementation of a UC\&C solution is not trivial. A key component and goal of this GM case study was the establishment of a comprehensive end- 
to-end framework for UC\&C design, deployment, and end user adoption. Many existing frameworks for IT design and deployment were reviewed, however, none were identified as having the required composite process and technology modules necessary to deliver an end-to-end strategy. Important to GM was the development of a framework that allowed for continued and controlled adaption, modification and streamlining of the UC\&C solution that could, over time, meet changing business needs. A framework was developed that incorporated IT governance, stakeholder and technical acceptance models [22] aligned to UC\&C deployment through the introduction of detailed implementation, service management, end user technical adoption plans and incorporated a sixlayer UC\&C technical reference architecture model [23]. The resulting framework is comprehensive in relation to meeting the planning, technical, program and end user governance required for initial deployment of a UC\&C solution as well as providing a lifecycle model for ongoing modification and development of new and improved functionality.

Further, research in the form of end user surveys and one-on-one interviews is being conducted within GM as a follow up to UC\&C deployment. Central to these studies is the analysis of end user experience and sentiment for the system, individual and group productivity, and end user behavior. Results of this research will be publicized during the first half of 2017. The data gained from these initiatives will be leveraged to correlate, validate, and further evaluate the findings inferred by the technical metrics and usage statistics collected during the months following deployment.

The vision of a UC\&C integrated digital enterprise provides an enticing view into the possible benefits offered to the digital enterprise, however the transformation to this end state is not simple. Success transcends the purely technical domains of infrastructure, communications, and applications and requires successful integration and acceptance of the associated human players, the people. Unlike networks built to support digital "things" for the purpose of data collection and digital analysis, a UC\&C framework must place "people" central to its goals. It is therefore essential that human considerations be taken into account when evaluating system needs and assessing end state success. Just as digitization promises to extend the benefits of digital and technical innovation to enrich people's lives, UC\&C additionally offers the potential to leverage the benefits of rich collaboration, interactive experience, and human intelligence to transform the workplace.

\section{References}

1] N. Burg, "How Technology has Changed Workplace Communication", Forbes/ Tech, Online: http://www.forbes.com/sites/unify/2013/12/10/howtechnology-has-changed-workplace-communication/print/, 2013.

[2] B. Elliot and S. Blood, "Magic Quadrant for Unified Communications", Gartner Research, 2015.

[3] Gartner, Inc., "Gartner Says Unified Communications Projects Should Focus on Increasing Business Agility, not Reducing IT Costs", Online: http://www.gartner.com/newsroom/id/654807, 2008.

[4] K. Riemer and S. Taing, "Unified Communications", Business \& Information Systems Engineering, 1(4), 2009, pp. 326-330.

[5] Grand Review Research, "Unified Communications Market Analysis by Product (On Premise, Cloudbased/Hosted), by Application (Education, Enterprise, Government, Healthcare) and Segment Forecasts to 2020", Online: http://www.grandviewresearch.com/industryanalysis/unified-communication-market, 2015.

[6] T. H. Davenport, "Thinking for a Living: How to get Better Performances and Results from Knowledge Workers", Harvard Business Press, 2013.

[7] F. Hussain, C. Lucas, and M. Ali, Managing Knowledge Effectively", Journal of Knowledge Management Practice, 5(1), 2004, pp. 1-12.

[8] International Telecommunications Union (ITU), "Measuring the Information Society", Online: www.itu.int/en/ITU-

D/Statistics/Documents/publications/mis2012/MIS2012_with wit_Annex_4.pdf, 2012.

[9] A. Sinan, B, Erik and V. A. Marshall, "Information, Technology, and Information Worker Productivity", Information Systems Research, 23(3), 2012, pp: 849-867.

[10] T. A. Carte, L. Chidambaram, and A. Becker, "Emergent Leadership in Self-managed Virtual Teams: A Longitudinal Study of Concentrated and Shared Leadership Behaviors", Group Decision and Negotiation, 15(4), 2006, pp. 323-343.

[11] L. Dube and G. Pare, "Global Virtual Teams", Communications of the ACM, 44(12), 2001, pp. 71-73.

[12] J. Greenough, "How the 'Internet of Things' will Impact Consumers, Businesses, and Governments in 2016 and Beyond", Business Insider. Online: http://www.businessinsider.com/how-the-internet-of-thingsmarket-will-grow-2014-10. 2015. 
[13] O. Vermesan, P. Friess, P. Guillemin, S. Gusmeroli, H. Sundmaeker, A. Bassi, I.S. Jubert, M. Mazura, M. Harrision, M. Eisenhaurer and P.Doody, "Internet of Things Strategic Research Roadmap", Global Technological and Societal Trends, 2011, pp. 9-52.

[14] I. Lazar, "Integrating Telephony, IM, Video and Mobility with Presence", Business Communications Review, 36(6), 2006.

[15] H. Lei and A. Ranganathan, "Context-aware Unified Communication", in Proceedings of the 2004 IEEE International Conference on Mobile Data Management, Berkeley, CA, 2003, pp. 176-186.

[16] M. Silic and A. Back, "Organizational Culture Impact on Acceptance and Use of Unified Communications \& Collaboration Technology in Organizations", in Proceedings of the BLED eConference, Bled, Slovenia, 2013.

[17] G. Pervan, D. Bajwa and L. F. Lewis, "A Study of the Adoption and Utilization of Seven Collaboration Technologies in Large Organizations in Australia and New Zealand", Journal of Global Information Technology Management, 8(2), 2005, pp. 5-26.

[18] C. Pickering and E. Wynn, "An Architecture and Business Process Framework for Global Team Collaboration", Intel Technology Journal, 8(4), 2004.
[19] S. Greengard, "Reaping the Benefits of Unified Communications", $\quad C I O \quad$ Insight, Online: http://www.cioinsight.com/it-strategy/messagingcollaboration/reaping-the-benefits-of-unifiedcommunications, 2013.

[20] M. Finneran, "Research: 2014 State of Unified Communications", Information Week, Online: http://reports.informationweek.com/abstract/9/12501/IPTelephony-Unified-Communications/Research:-2014-Stateof-Unified-Communications.html, 2014.

[21] G. C. Kane, D. Palmer, A. N. Phillips, D. Kiron and N. Buckley, "Strategy, not Technology, Drives Digital Transformation. MIT Sloan Management Review and Deloitte University Press, 2015.

[22] O. Jokonya, J. H. Kroeze, and J. A. van der Poll, "Towards a Framework for Decision Making Regarding IT Adoption", in Proceedings of the South African Institute for Computer Scientists and Information Technologists Conference, ACM. 2015, pp. 316-325.

[23] A. Bolton, L. Goosen, and E. Kritzinger, "Enterprise Digitization Enablement through Unified Communication \& Collaboration", in Proceedings of the South African Institute for Computer Scientists and Information Technologists Conference, ACM. 2016. 